\title{
Existence of non-uniform cocycles on uniquely ergodic systems
}

\author{
Daniel Lenz ${ }^{1}$ \\ Fakultät für Mathematik, TU Chemnitz, 09107 Chemnitz, Germany
}

Received 5 June 2002; accepted 15 April 2003

\begin{abstract}
We study existence of non-uniform continuous $S L(2, \mathbb{R})$-valued cocycles over uniquely ergodic dynamical systems. We present a class of subshifts over finite alphabets on which every locally constant cocycle is uniform. On the other hand, we also show that every irrational rotation admits non-uniform cocycles. Finally, we discuss characterizations of uniformity. (c) 2003 Elsevier SAS. All rights reserved.

\section{Résumé}

On étudie l'existence de cocycles non uniformes à valeurs dans $S L(2, \mathbb{R})$, pour les systèmes dynamiques uniquement ergodiques. On présente une classe de sous-shifts à alphabets finis pour lesquels tout cocycle localement constant est uniforme. Par ailleurs, on montre que toute rotation irrationelle admet des cocycles non-uniformes. Enfin, nous présentons des caractérisations de l'uniformité.

(c) 2003 Elsevier SAS. All rights reserved.
\end{abstract}

MSC: $37 \mathrm{H} 15 ; 47 \mathrm{~B} 80$

Keywords: Ergodic theorem; Uniform cocycle; Hyperbolicity

\section{Introduction}

This paper is concerned with $\operatorname{SL}(2, \mathbb{R})$-valued cocycles over dynamical systems. Throughout, $(\Omega, T)$ will be a uniquely ergodic dynamical system (i.e. $\Omega$ is a compact metric space, $T: \Omega \rightarrow \Omega$ is a homeomorphism and there is only one $T$-invariant probability measure on $\Omega$ ). The unique $T$-invariant probability measure on $\Omega$ will be denoted by $\mu$. Let $S L(2, \mathbb{R})$ denote the group of real-valued $2 \times 2$-matrices with determinant equal to one. This is a topological group whose topology is induced by the standard metric on the $2 \times 2$-matrices. To a continuous function $A: \Omega \rightarrow S L(2, \mathbb{R})$ we associate the cocycle

$$
A(\cdot, \cdot): \mathbb{Z} \times \Omega \rightarrow S L(2, \mathbb{R})
$$

\footnotetext{
E-mail address: dlenz@mathematik.tu-chemnitz.de (D. Lenz).

1 This research was supported in part by THE ISRAEL SCIENCE FOUNDATION (grant no. 447/99) and by the Edmund Landau Center for Research in Mathematical Analysis and Related Areas, sponsored by the Minerva Foundation (Germany). 
defined by

$$
A(n, \omega) \equiv \begin{cases}A\left(T^{n-1} \omega\right) \cdots A(\omega), & n>0 \\ I d, & n=0 \\ A^{-1}\left(T^{n} \omega\right) \cdots A^{-1}\left(T^{-1} \omega\right), & n<0 .\end{cases}
$$

By the multiplicative ergodic theorem, there exists a $\Lambda(A) \in \mathbb{R}$ with

$$
\Lambda(A)=\lim _{n \rightarrow \infty} \frac{1}{n} \log \|A(n, \omega)\|
$$

for $\mu$-almost every $\omega \in \Omega$. Following [6] (cf. [21] as well), we introduce the following definition.

Definition 1. Let $(\Omega, T)$ be uniquely ergodic. The continuous function $A: \Omega \rightarrow S L(2, \mathbb{R})$ is called uniform if the limit $\Lambda(A)=\lim _{n \rightarrow \infty} \frac{1}{n} \log \|A(n, \omega)\|$ exists for all $\omega \in \Omega$ and the convergence is uniform on $\Omega$.

Remark 1. For minimal (not necessarily uniquely ergodic) systems, uniform existence of the limit in the definition already implies uniform convergence, as proven by Furstenberg and Weiss [7]. Their result is actually even more general and applies to arbitrary real valued continuous cocycles.

Existence or non-existence of uniform $S L(2, \mathbb{R})$-valued functions has been studied by various people, e.g. in $[21,8,6,16]$. In fact, Walters asked the following question [21]:

(Q) Does every uniquely ergodic dynamical system with non-atomic measure $\mu$ admit a non-uniform cocycle?

Using results of Veech [20], Walters presents a class of examples admitting non-uniform cocycles. He also discusses a further class of examples, namely suitable irrational rotations, for which non-uniformity was shown by Herman [8]. Recently, Furman carried out a careful study of uniformity of cocycles [6]. For strictly ergodic dynamical systems, he characterizes uniform cocycles with positive $\Lambda(A)$ in terms of uniform diagonalizability. Related results on positivity of cocycles can also be found in [12].

The aim of this article is to adress (Q) for certain examples and to study conditions for uniformity of cocycles. In order to be more precise recall that $(\Omega, T)$ is called a subshift over the compact $S$, if $\Omega$ is a closed subset of $S^{\mathbb{Z}}$ (with product topology) invariant under the shift $T: S^{\mathbb{Z}} \rightarrow S^{\mathbb{Z}},(T s)(n) \equiv s(n+1)$. If $S$ is finite, it is called the alphabet. A function $f$ on a subshift over $S$ is called locally constant if there exists an $N \in \mathbb{N}$ such that

$$
f(\omega)=f(\rho), \quad \text { whenever }(\omega(-N), \ldots, \omega(N))=(\rho(-N), \ldots, \rho(N)) .
$$

Our results will show the following:

- There exist subshifts over finite alphabets which do not admit locally constant non-uniform cocycles (Theorem 1).

- Every irrational rotation admits a non-uniform cocycle (Theorem 2).

- For strictly ergodic dynamical systems, uniformity of $A$ with $\Lambda(A)>0$ follows already from suitable lower bounds on $n^{-1} \ln \|A(n, \omega)\|$ (Theorem 3$)$.

- For uniquely ergodic dynamical system, uniformity of $A$ with $\Lambda(A)>0$ can be characterized by a certain uniform hyperbolicity condition (Theorem 4).

As mentioned already, these results are closely related to results of Furman [6] and Herman [8,9] respectively. This will be discussed in more detail at the corresponding places.

This paper is organised as follows. In Section 2, we prove Theorem 1. Section 3 is devoted to a proof of Theorem 2 and discussion of its background. Finally, we discuss Theorem 3 and Theorem 4 in Section 4. 


\section{Subshifts with only uniform locally constant functions}

In this section we present a class of subshifts over finite alphabets on which every locally constant cocycle is uniform.

For a subshift $(\Omega, T)$ over the finite set $S$, let $\mathcal{W}$ be the associated set of finite words i.e.

$$
\mathcal{W} \equiv\left\{\omega(n) \ldots \omega(n+k): \omega \in \Omega, n \in \mathbb{Z}, k \in \mathbb{N}_{0}\right\} .
$$

We will use standard concepts from combinatorics on words. In particular, we define the length $|w|$ of a word $w=w(1) \ldots w(n)$ to be $n$ and we denote the number of copies of $v$ in $w$ by $\sharp_{v}(w)$ for arbitrary $v, w \in \mathcal{W}$. The class of subshifts we are particularly interested in is presented in the next definition.

Definition 2. A subshift $(\Omega, T)$ over the finite set $S$ is said to satisfy uniform positivity of weights, (PW), if there exists a constant $C>0$ with $\liminf _{|w| \rightarrow \infty} \frac{\sharp_{v}(w)}{|w|}|v| \geqslant C$ for all $v \in \mathcal{W}$.

Remark 2. (a) Condition (PW) says roughly that the amount of "space" covered by a word $v \in \mathcal{W}$ in a long word $w \in \mathcal{W}$ is bounded below uniformly in $v \in \mathcal{W}$. In particular, (PW) implies minimality.

(b) The condition (PW) is in particular satisfied for subshifts associated to primitive substitutions and more generally for linearly recurrent subshifts [5,17].

(c) It is not hard to see that (PW) implies that the subshift has linear complexity. More precisely, the number of different words in $\mathcal{W}$ of length $n$ is bounded by $C^{-1} n$ (see e.g. [18]).

Theorem 1. Let $(\Omega, T)$ be a subshift over the finite set $S$. If $(\Omega, T)$ satisfies $(P W)$, then every locally constant function $G: \Omega \rightarrow S L(2, \mathbb{R})$ is uniform.

The theorem is a rather direct consequence of the following lemma. The lemma relates (PW) to existence of averages for subadditive functions on $\mathcal{W}$. Recall that $F: \mathcal{W} \rightarrow \mathbb{R}$ is called subadditive if $F(x y) \leqslant F(x)+F(y)$ for arbitrary $x, y \in \mathcal{W}$ with $x y \in \mathcal{W}$.

Lemma 2.1. Let $(\Omega, T)$ be a minimal subshift over the finite $S$. Then, the limit $\lim _{|x| \rightarrow \infty}|x|^{-1} F(x)$ exists for every subadditive $F: \mathcal{W} \rightarrow \mathbb{R}$ if and only if $(\Omega, T)$ satisfies $(P W)$.

Proof. One implication follows from Theorem 2 of [17] and the other by Proposition 4.2. of [17].

Proof of Theorem 1. Define $F^{G}: \mathcal{W} \rightarrow \mathbb{R}$ by

$$
F^{G}(x) \equiv \sup \{\log \|G(|x|, \omega)\|: \omega(1) \ldots \omega(|x|)=x\} .
$$

Apparently, $F^{G}$ is subadditive. Thus, by the preceeding lemma, the limit $\lim _{|x| \rightarrow \infty}|x|^{-1} F^{G}(x)$ exists. Therefore, it remains to show that

$$
\Delta(n, \sigma, \rho) \equiv\left|\frac{1}{n} \log \|G(n, \sigma)\|-\frac{1}{n} \log \|G(n, \rho)\|\right|
$$

is arbitrarily small for all $\sigma, \rho \in \Omega$ with

$$
\sigma(1) \ldots \sigma(n)=\rho(1) \ldots \rho(n)
$$

whenever $n \in \mathbb{N}$ is large enough. Let $N \in \mathbb{N}$ be the constant of (1) for the locally constant $G$. Consider an arbitrary $n \in \mathbb{N}$ with $n \geqslant 2 N$.

From $G(n, \omega)=G\left(N, T^{n-N} \omega\right) G\left(n-2 N, T^{N} \omega\right) G(N, \omega)$ for arbitrary $n \geqslant 2 N$, we infer

$$
\log \|G(n, \omega)\| \leqslant \log \left\|G\left(n-2 N, T^{N} \omega\right)\right\|+\log \left\|G\left(N, T^{n-N} \omega\right)\right\|+\log \|G(N, \omega)\|
$$


as well as $G\left(n-2 N, T^{N} \omega\right)=G\left(N, T^{n-N} \omega\right)^{-1} G(n, \omega) G(N, \omega)^{-1}$ for arbitrary $\omega \in \Omega$. Combining this latter equality with the fact that $\|M\|=\left\|M^{-1}\right\|$ for all $M \in S L(2, \mathbb{R})$, we infer

$$
\log \left\|G\left(n-2 N, T^{N} \omega\right)\right\|-\log \left\|G\left(N, T^{n-N} \omega\right)\right\|-\log \|G(N, \omega)\| \leqslant \log \|G(n, \omega)\|
$$

for all $\omega \in \Omega$. By local constancy, we have $G\left(n-2 N, T^{N} \sigma\right)=G\left(n-2 N, T^{N} \rho\right)$ whenever $\sigma$ and $\rho$ satisfy (3) with $n \geqslant 2 N$. Thus, for such $\sigma, \rho$ the above inequalities yield

$$
|\log \|G(n, \sigma)\|-\log \|G(n, \rho)\|| \leqslant 4 \sup \{|\log \|G(N, \omega)\||: \omega \in \Omega\} .
$$

As the right hand side is independent of $n$, this easily gives the desired smallness of the $\Delta(n, \sigma, \rho)$ in $(2)$ for large $n$.

\section{Non-uniform functions}

In this section we will discuss certain examples of non-uniform cocycles. These examples will be based on recent results of the author [16] on spectral theory of certain Schrödinger operators and known results on positivity of Lyapunov exponents [1,2,9].

Let $(\Omega, T)$ be as above and let $f: \Omega \rightarrow \mathbb{R}$ be a continuous function. To these data we can associate a family $\left(H_{\omega}\right)_{\omega \in \Omega}$ of operators $H_{\omega}: \ell^{2}(\mathbb{Z}) \rightarrow \ell^{2}(\mathbb{Z}), \omega \in \Omega$, given by

$$
\left(H_{\omega} u\right)(n) \equiv u(n+1)+u(n-1)+f\left(T^{n-1} \omega\right) u(n) .
$$

Such families of operators arise in the study of disordered media. Depending on the underlying dynamical systems, they provide examples for a variety of interesting spectral features such as dense pure point spectrum, purely singularly continuous spectrum and Cantor spectrum of measure zero (see [3,4] for details and further references).

An important tool in the investigation of their spectral theory is the study of solutions $u$ of the associated eigenvalue equation

$$
u(n+1)+u(n-1)+(\omega(n)-E) u(n)=0
$$

for $E \in \mathbb{R}$. It is not hard to see that $u$ is a solution of this equation if and only if

$$
\left(\begin{array}{c}
u(n+1) \\
u(n)
\end{array}\right)=M^{E}(n, \omega)\left(\begin{array}{l}
u(1) \\
u(0)
\end{array}\right), \quad n \in \mathbb{Z},
$$

where the continuous function $M^{E}: \Omega \rightarrow S L(2, \mathbb{R})$ is defined by

$$
M^{E}(\omega) \equiv\left(\begin{array}{cc}
E-f(\omega) & -1 \\
1 & 0
\end{array}\right) .
$$

As discussed in the introduction $M^{E}$ gives rise to the average $\gamma(E) \equiv \Lambda\left(M^{E}\right)$. This average is called the Lyapunov exponent for the energy $E$. It measures the rate of exponential growth of solutions of (5).

As is well known (see e.g. Proposition 1.2.2 in [15]), for minimal $(\Omega, T)$ the spectrum $\Sigma=\sigma\left(H_{\omega}\right)$ of the selfadjoint operator $H_{\omega}$ does not depend on the point $\omega \in \Omega$. Moreover, for strictly ergodic systems, it was shown by the author in Theorem 3 of [16] that

$$
\Sigma=\{E: \gamma(E)=0\} \cup\left\{E: M^{E} \text { is not uniform }\right\},
$$

where the union is disjoint. This implies immediately the following result.

Lemma 3.1. Let $(\Omega, T)$ be strictly ergodic and $\left(H_{\omega}\right)$ as above. Then $\Sigma=\left\{E: M^{E}\right.$ is not uniform $\}$ if and only if $\gamma(E)>0$ for every $E \in \mathbb{R}$. 
Thus, examples of operators of the form $\left(H_{\omega}\right)$ with positive Lyapunov exponent give rise to non-uniform matrices. Indeed, there are well known examples of operators with uniformly positive Lyapunov exponent and we will discuss one of them next.

Fix $\alpha \in(0,1)$ irrational and $\lambda>0$. Denote the irrational rotation by $\alpha$ on the unit circle, $\mathcal{S}$, by $R_{\alpha}$ (i.e. $R_{\alpha} z \equiv \exp (2 \pi i \alpha) z$, where $i$ is the square root of -1$)$. Define $f^{\lambda}: \mathcal{S} \rightarrow \mathbb{R}$ by $f^{\lambda}(z) \equiv \lambda\left(z+z^{-1}\right)$ (i.e. $\left.f^{\lambda}(\exp (i \theta))=2 \lambda \cos (\theta)\right)$. Denote the associated operators by $\left(H_{z}^{\lambda}\right)$ and their spectrum by $\Sigma(\lambda)$. The operators $\left(H_{z}^{\lambda}\right)$ are called almost-Mathieu operators. They have attracted much attention (see e.g. [11,10,13] for further discussion and references). We have the following theorem.

Theorem 2. For arbitrary irrational $\alpha \in(0,1)$ and $\lambda>1$, the function $M^{E}$ is non-uniform if and only if $E$ belongs to $\Sigma(\lambda)$.

Proof. By the foregoing lemma, it suffices to show positivity of $\gamma(E)$ for every $E \in \mathbb{R}$. This is well known [1,2] (see [9] for an alternative proof as well).

Remark 3. The result shows that every irrational rotation allows for a non-uniform matrix. This generalizes results of Herman [8], where this was only shown for certain rotation numbers. Note, however, that the results of [9] combined with Theorem 4 of [6] (or Theorem 4 below) also show existence of non-uniform cocycles for every irrational rotation. Still, the above result is more explicit in that the set of energies with non-uniform matrices is identified as $\Sigma(\lambda)$.

\section{Characterizations of uniformity}

In this section we study uniformity of cocycles for uniquely ergodic and strictly ergodic systems.

Let $\mathcal{P}=\mathcal{P} \mathbb{R}^{2}$ be the projective space over $\mathbb{R}^{2}$. Thus, $\mathcal{P}$ is the space of all one-dimensional subspaces of $\mathbb{R}^{2}$. To $X \in \mathbb{R}^{2} \backslash\{0\}$, we associate the element $[X]=\{\lambda X: \lambda \in \mathbb{R}\} \in \mathcal{P}$. Obviously, every element in $\mathcal{P}$ can be written as $[(\cos (\theta), \sin (\theta))]$ with a suitable $\theta \in[0, \pi]$. The space $\mathcal{P}$ is a complete metric space, when equipped with the metric

$$
d([(\cos (\theta), \sin (\theta))],[(\cos (\eta), \sin (\eta))])=\min \{|\theta-\eta|,|\theta-\eta-\pi|,|\theta-\eta+\pi|\} .
$$

We start with a characterization of uniformity of cocycles for strictly ergodic systems.

Theorem 3. Let $(\Omega, T)$ be strictly ergodic. Then, a continuous $A: \Omega \rightarrow S L(2, \mathbb{R})$ is uniform with $\Lambda(A)>0$ if and only if there exist $m \in \mathbb{N}$ and $\delta>0$ such that $\delta \leqslant \frac{1}{n} \ln \|A(n, \omega)\|$ for all $\omega \in \Omega$ and $n \geqslant m$.

Remark 4. The theorem deals with a uniform lower bound on $\frac{1}{n} \ln \|A(n, \omega)\|$. As for an upper bound, we mention Corollary 2 of [6] which shows $\limsup _{n \rightarrow \infty} n^{-1} \ln \|A(n, \omega)\| \leqslant \Lambda(A)$ uniformly in $\omega \in \Omega$ for arbitrary (not necessarily uniform) continuous $A: \Omega \rightarrow S L(2, \mathbb{R})$.

The proof of this theorem and of further results will be based on some auxiliary propositions.

Proposition 4.1. Let $\left(A_{n}\right)$ be a sequence in $S L(2, \mathbb{R})$. Then, there exists at most one $v \in \mathcal{P}$ with $\left\|A_{n} V\right\| \rightarrow 0$, $n \rightarrow \infty$, for every $V \in v$.

Proof. Assume the contrary. Then, there exist linearly independent vectors $V_{1}$ and $V_{2}$ in $\mathbb{R}^{2}$ with $\left\|A_{n} V_{i}\right\| \rightarrow 0$, $n \rightarrow \infty, i=1,2$. Thus, $\|A\| \rightarrow 0, n \rightarrow 0$ and this contradicts $\left\|A_{n}\right\| \geqslant 1$ (which is a direct consequence of $\left.\operatorname{det} A_{n}=1\right)$. 
Part (a) of the following proposition contains the key to our considerations, viz the estimate (10) below. We take it from recent work of Last/Simon in [14] which in turn essentially abstracts a result of Ruelle [19]. As pointed out to the author by the referee it can also be understood as a consequence of the classical geometric Morse-Lemma by viewing $S L(2, \mathbb{R})$ as the group of isometries of the hyperbolic plane and then using that the orbit in question $V_{n}=A_{n}^{-1} V_{0}$ is quasi-geodesic (due to the assumptions).

While (a) of the proposition is clearly the main new input in our argument, we will mostly use the the variant of (a) given in part (b) of the proposition.

Proposition 4.2. Let $\left(A_{n}\right)$ be a sequence of matrices in $\operatorname{SL}(2, \mathbb{R})$ with $D \equiv \sup _{n \in \mathbb{N}}\left\|A_{n+1} A_{n}^{-1}\right\|<\infty$. Define the selfadjoint operator $\left|A_{n}\right|$ by $\left|A_{n}\right| \equiv\left(A_{n}^{*} A_{n}\right)^{1 / 2}$ and let $u_{n}$ be the eigenspace of $\left|A_{n}\right|$ associated to the eigenvalue $a_{n} \equiv\left\|\left|A_{n}\right|\right\|^{-1}=\left\|A_{n}\right\|^{-1}$.

(a) If there exist $\delta>0$ and $m \in \mathbb{N}$ with $\delta \leqslant n^{-1} \ln \left\|A_{n}\right\|$ for $n \geqslant m$ then $u_{n}$ is one-dimensional for $n \geqslant m$ i.e. $u_{n} \in \mathcal{P}$, and there exists $u \in \mathcal{P}$ with $d\left(u_{n}, u\right) \leqslant C \exp (-2 \delta n)$ for every $n \geqslant m$, where $C=2 \pi D^{2}(1-\exp (-2 \delta))^{-1}$.

(b) If there exist $\delta>0$ and $m \in \mathbb{N}$ with $\delta \leqslant n^{-1} \ln \left\|A_{n}\right\| \leqslant \frac{3}{2} \delta$ for $n \geqslant m$, then $\left\|A_{n} U\right\| \leqslant(2 C+1) \times$ $\exp \left(-2^{-1} \delta n\right)\|U\|$ for arbitrary $n \geqslant m$ and $U \in u$.

Proof. (a) As $\left|A_{n}\right|$ is selfadjoint, $a_{n}^{-1}=\left\|\left|A_{n}\right|\right\|$ is an eigenvalue of $\left|A_{n}\right|$. Thus, by $1=\operatorname{det} A_{n}=\operatorname{det}\left|A_{N}\right|$, the selfadjoint $\left|A_{n}\right|$ has the eigenvalues $a_{n}^{-1}$ and $a_{n}$. As by assumption

$$
1<\exp (\delta n) \leqslant\left\|A_{n}\right\|=a_{n}^{-1} \text { for all } n \geqslant m .
$$

the eigenspace $u_{n}$ is then one-dimensional. By (8.5) of [14] (see [19] as well), the $u_{n}$ converge to an element $u \in \mathcal{P}$ and

$$
d\left(u_{n}, u\right) \leqslant \frac{\pi}{2} \sum_{k=n}^{\infty} \frac{D^{2}}{\left\|A_{n}\right\|^{2}} .
$$

Combining (9) and (10), we infer

$$
d\left(u_{n}, u\right) \leqslant C \exp (-2 \delta n)
$$

with $C$ as above.

(b) Let $U \in u$ with $\|U\|=1$ and $n \geqslant m$ be given. By (11), we can find $U_{n} \in u_{n}$ with $\left\|U_{n}\right\|=1$ and

$$
\left\|U-U_{n}\right\| \leqslant \sqrt{2} d\left([U],\left[U_{n}\right]\right) \leqslant C \sqrt{2} \exp (-2 \delta n) .
$$

By (9) we have

$$
\left\|A_{n} U_{n}\right\|=\left\|\left|A_{n}\right| U_{n}\right\|=\left\|a_{n} U_{n}\right\| \leqslant \exp (-\delta n) .
$$

As, by assumption, $\ln \left\|A_{n}\right\| \leqslant \frac{3}{2} \delta n$, we obtain

$$
\left\|A_{n} U\right\| \leqslant\left\|A_{n}\left(U-U_{n}\right)\right\|+\left\|A_{n} U_{n}\right\| \leqslant(2 C+1) \exp \left(-\frac{1}{2} \delta n\right) .
$$

This implies (b).

We also have the following "uniform version" of the foregoing proposition.

Proposition 4.3. Let $A: \Omega \rightarrow S L(2, \mathbb{R})$ be continuous. For $n \in \mathbb{Z}$ and $\omega \in \Omega$, define the selfadjoint nonnegative operator $|A(n, \omega)|$ by $|A(n, \omega)|=\left(A(n, \omega)^{*} A(n, \omega)\right)^{1 / 2}$ and let $u(n, \omega)$ be the eigenspace of $|A(n, \omega)|$ associated to the eigenvalue $a(n, \omega)=\|A(n, \omega)\|^{-1}=\||A(n, \omega)|\|^{-1}$. 
(a) If there exist $\delta>0$ and $m \in \mathbb{N}$ with $\delta \leqslant n^{-1} \ln \|A(n, \omega)\|$ for every $n \geqslant m$ and every $\omega \in \Omega$, then $u(n, \omega)$ is one-dimensional, i.e. $u(n, \omega)$ belongs to $\mathcal{P}$, for $n \geqslant m$ and the functions $u(n, \cdot)$ converge uniformly to a continuous function $u: \Omega \rightarrow \mathcal{P}$.

(b) If there exist $\delta>0$ and $m \in \mathbb{N}$ with $\delta \leqslant n^{-1} \ln \|A(n, \omega)\| \leqslant \frac{3}{2} \delta$, for all $\omega \in \Omega$ and $n \geqslant m$, then there exists $\kappa>0$ and $C>0$ with $\|A(n, \omega) U\| \leqslant C \exp (-\kappa n)\|U\|$ for every $n \in \mathbb{N}, \omega \in \Omega$ and $U \in u(\omega)$.

Proof. To prove (a) and (b), we apply parts (a) and (b) respectively of the foregoing proposition simultanuously for all $\omega \in \Omega$. Note that all estimates in the foregoing proposition are rather explicit and are governed by constants not depending on $\omega \in \Omega$. In particular, the functions $u(n, \cdot)$ converge uniformly. As they are obviously continuous, their limit is also continuous.

Proof of Theorem 3. The "only if" statement is clear. To show the other direction, we proceed as follows:

By assumption we can apply Proposition 4.3(a) and obtain a continuous function $u: \Omega \rightarrow \mathcal{P}$ (which is the limit of the function $u(n, \cdot))$. By the multiplicative ergodic theorem, there exists a $T$-invariant set $\Omega^{\prime} \subset \Omega$ of full measure with

$$
0<\delta \leqslant \Lambda(A)=\liminf _{|n| \rightarrow \infty} \frac{1}{|n|} \ln \|A(n, \omega)\|=\limsup _{|n| \rightarrow \infty} \frac{1}{|n|} \ln \|A(n, \omega)\|
$$

for every $\omega \in \Omega^{\prime}$. This, of course, implies

$$
0<\Lambda(A) \leqslant \limsup _{n \rightarrow \infty} \frac{1}{n} \ln \|A(n, \omega)\| \leqslant \frac{4}{3} \liminf _{n \rightarrow \infty} \frac{1}{n} \ln \|A(n, \omega)\|
$$

for every $\omega \in \Omega^{\prime}$. By (b) of Proposition 4.2, we then infer exponential decay of $\|A(n, \omega) U\|$ for $n \rightarrow \infty$ for arbitrary but fixed $\omega \in \Omega^{\prime}$ and $U \in u(\omega)$. As $\Omega^{\prime}$ is invariant and the subspace of $\mathbb{R}^{2}$ with such exponential decay is unique by Proposition 4.1, we conclude, for $\omega \in \Omega^{\prime}$,

$$
[A(n, \omega) U]=u\left(T^{n} \omega\right)
$$

for $n \in \mathbb{Z}$ and $U \in u(\omega) \backslash\{0\}$. Now, by continuity of $\omega \mapsto u(\omega)$ and minimality of $(\Omega, T)$, we infer validity of (14) for every $\omega \in \Omega$ and $n \in \mathbb{Z}$. Similarly, considering $n \rightarrow-\infty$, we infer existence of a continuous $v: \Omega \rightarrow \mathcal{P}$, $\omega \mapsto v(\omega)$, such that $\|A(n, \omega) V\|$ is exponentially decaying for $n \rightarrow-\infty$ for every $\omega \in \Omega^{\prime}$ and $V \in v(\omega)$ and

$$
[A(n, \omega) V]=v\left(T^{n} \omega\right)
$$

for arbitrary $\omega \in \Omega, n \in \mathbb{Z}$ and $V \in v(\omega) \backslash\{0\}$.

Now, choose, for each $\omega \in \Omega$, vectors $U(\omega) \in u(\omega)$ and $V(\omega) \in v(\omega)$ with $\|U(\omega)\|=\|V(\omega)\|=1$. By (14) and (15), there exist $a, d: \Omega \rightarrow \mathbb{R} \backslash\{0\}$, with $A(\omega) U(\omega)=a(\omega) U(T \omega)$ and $A(\omega) V(\omega)=d(\omega) V(T \omega)$. Define the matrix $C(\omega)$ by $C(\omega)=(U(\omega), V(\omega))$. By $\|U(\omega)\|=\|V(\omega)\|=1, U(\omega)$ and $V(\omega)$ are unique up to a multiplication by -1 . Moreover, for fixed $\omega_{0} \in \Omega$, we can always find a neighbourhood of $\omega_{0}$ on which $U$ and $V$ can be chosen continuously (as $u$ and $v$ are continuous). Therefore, the functions

$$
\omega \mapsto\|C(\omega)\|, \quad \omega \mapsto|a(\omega)|, \quad \omega \mapsto|d(\omega)|
$$

are continuous (as they are invariant under the replacement of $U(\omega)$ by $-U(\omega)$ or $V(\omega)$ by $-V(\omega)$.) A short calculation then gives

$$
\ln \|A(n, \omega) U(\omega)\|= \begin{cases}\sum_{k=0}^{n-1} \ln \left|a\left(T^{k} \omega\right)\right|, & n>0, \\ 0, & n=0, \\ -\sum_{k=n}^{-1} \ln \left|a\left(T^{k} \omega\right)\right|, & n<0 .\end{cases}
$$

Thus, the uniform ergodic theorem for continuous functions on uniquely ergodic systems, yields

$$
\frac{1}{n} \ln \|A(n, \omega) U(\omega)\| \rightarrow \int_{\Omega} \ln |a(\omega)| d \mu(\omega), \quad|n| \rightarrow \infty,
$$


uniformly in $\omega \in \Omega$. As $\|A(n, \omega) U(\omega)\|$ is exponentially decaying for $n \rightarrow \infty$ and $\omega \in \Omega^{\prime}$, we see $\int_{\Omega} \ln |a(\omega)| d \mu(\omega)<0$. Putting this together, we infer that $\|A(n, \omega) U(\omega)\|$ is exponentially decaying for $n \rightarrow \infty$ and exponentially increasing for $n \rightarrow-\infty$ for every $\omega \in \Omega$. Similarly, $\|A(n, \omega) V(\omega)\|$ can be seen to be exponentially decaying for $n \rightarrow-\infty$ and exponentially increasing for $n \rightarrow \infty$ for every $\omega \in \Omega$. In particular, we have $u(\omega) \neq v(\omega)$ for every $\omega \in \Omega$. Thus, the matrix $C(\omega)$ is invertible and, by construction, we have

$$
C(T \omega)^{-1} A(\omega) C(\omega)=\left(\begin{array}{cc}
a(\omega) & 0 \\
0 & d(\omega)
\end{array}\right) .
$$

Now, uniformity of $A$ follows easily from continuity of $|a|$ and $|b|$, as the continuous functions $\omega \mapsto\|C(\omega)\|$ and $\omega \mapsto\left\|C^{-1}(\omega)\right\|$ are uniformly bounded on the compact $\Omega$.

Corollary 4.4. Let $(\Omega, T)$ be strictly ergodic and $\left(H_{\omega}\right)_{\omega \in \Omega}$ as in Section 3. For $E \in \mathbb{R}$, define $\gamma_{\min }(E)$ by $\gamma_{\min }(E) \equiv \liminf _{n \rightarrow \infty} \min \left\{\frac{1}{n} \ln \left\|M^{E}(n, \omega)\right\|: \omega \in \Omega\right\}$. Then, $\Sigma=\left\{E \in \mathbb{R}: \gamma_{\min }(E)=0\right\}$.

Proof. By Theorem 3, we have $\gamma_{\min }(E)>0$ if and only if $M^{E}$ is uniform with $\gamma(E)>0$. But this is equivalent to $E \notin \Sigma$ by (8).

Remark 5. For the almost-Mathieu operators discussed in Section 3, it is possible to establish pure point spectrum (provided $\alpha, \lambda$ are suitable) (see references in Section 3). An important issue in the corresponding proofs is to obtain exponentially growing lower bounds on the modulus of the matrix elements of $M^{E}(n, \omega)$ for large $n \in \mathbb{N}$ (and suitable $\omega \in \Omega$ and $E \in \mathbb{R}$ ). The corollary shows that these bounds can not hold uniformly. This contrasts with the validity of uniform upper bounds discussed in Remark 4.

The methods developed above to treat strictly ergodic systems can be modified to characterize uniformity of cocylces for uniquely ergodic systems. This is the content of the next theorem.

Theorem 4. Let $(\Omega, T)$ be uniquely ergodic and $A: \Omega \rightarrow S L(2, \mathbb{R})$ be continuous. Then the following are equivalent:

(i) A is uniform with $\Lambda(A)>0$.

(ii) There exist constants $\kappa, C>0$ and continuous functions $u, v: \Omega \rightarrow \mathcal{P}$ with

$$
\|A(n, \omega) U\| \leqslant C \exp (-\kappa n)\|U\| \text { and }\|A(-n, \omega) V\| \leqslant C \exp (-\kappa n)\|V\|
$$

for arbitrary $\omega \in \Omega, n \in \mathbb{N}, U \in u(\omega)$ and $V \in v(\omega)$.

(iii) There exists $\delta>0$ and $m \in \mathbb{N}$ with $0<\delta \leqslant \frac{1}{n} \ln \|A(n, \omega)\| \leqslant \frac{3}{2} \delta$ for every $\omega \in \Omega$ and $n \geqslant m$.

In this case, $u(\omega) \neq v(\omega)$, $[A(n, \omega) U]=u\left(T^{n} \omega\right)$ and $[A(n, \omega) V]=v\left(T^{n} \omega\right)$ for arbitrary $\omega \in \Omega, n \in \mathbb{Z}, U \in$ $u(\omega)$ and $V \in v(\omega)$ with $U, V \neq 0$.

Remark 6. The equivalence of (i) and (ii) in some sense extends the corresponding result of Furman for strictly ergodic systems [6]. Namely, Theorem 4 of [6] shows that uniformity of $A$ combined with $\Lambda(A)>0$ holds if and only if $A$ is continuously cohomologous to a diagonal matrix. Our extension to uniquely ergodic systems is made possible through the use of Proposition 4.2 (see discussion before this proposition). Let us also mention that the concept of hyperbolic structure studied in [9] essentially amounts to (ii) in our context (see [8] for connection to uniformity as well). Part (iii) of Theorem 4 is new. It is inspired by arguments in [14]. It provides an analogue of Theorem 3 for uniquely ergodic systems.

Proof of Theorem 4. (i) $\Rightarrow$ (iii): This is clear.

(iii) $\Rightarrow$ (ii): The construction of $u$ is immediate from Proposition 4.3. The construction of $v$ is similar by applying Proposition 4.3 to the function $\widetilde{A}: \widetilde{\Omega} \rightarrow S L(2, \mathbb{R})$, where $\widetilde{\Omega}=\Omega, \widetilde{A}(\omega)=A\left(T^{-1} \omega\right)^{-1}$ and the action on $\widetilde{\Omega}$ is given by $\widetilde{T}=T^{-1}$. 
(ii) $\Rightarrow$ (i): Proposition 4.1 and assumption (ii) imply

$$
[A(n, \omega) U]=u\left(T^{n} \omega\right) \text { and }[A(n, \omega) V]=v\left(T^{n} \omega\right)
$$

for arbitrary $\omega \in \Omega, n \in \mathbb{Z}, U \in u(\omega)$ and $V \in v(\omega)$ with $U, V \neq 0$. Let arbitrary $U \in u(\omega)$ and $n \in \mathbb{N}$ be given. By (18) and (ii), we then have $\|U\|=\left\|A\left(n, T^{-n} \omega\right) A(-n, \omega) U\right\| \leqslant C \exp (-\kappa n)\|A(-n, \omega) U\|$ which implies $\|A(-n, \omega) U\| \geqslant C^{-1} \exp (\kappa n)\|U\|$. As this holds for all $n \in \mathbb{N}$, we infer $u(\omega) \neq v(\omega)$ from (ii). Now, (i) follows by mimicking the last part of the proof of Theorem 3 .

Note that the last statement of the theorem has been shown in (ii) $\Rightarrow$ (i).

To formulate our last result, we recall that the set $C(\Omega, S L(2, \mathbb{R}))$ of continuous functions $A: \Omega \rightarrow S L(2, \mathbb{R})$ is a complete metric space when equiped with the metric

$$
d\left(A_{1}, A_{2}\right) \equiv \sup _{\omega \in \Omega}\left\|A_{1}(\omega)-A_{2}(\omega)\right\| .
$$

Let $\mathcal{U}(\Omega)$ be the set of uniform $A \in C(\Omega, S L(2, \mathbb{R}))$ and $\mathcal{U}(\Omega)_{+}$be the set of those $A \in \mathcal{U}(\Omega)$ with $\Lambda(A)>0$. Then the following holds (see Theorem 5 of [6] as well).

Theorem 5. Let $(\Omega, T)$ be uniquely ergodic. Then, $\mathcal{U}(\Omega)_{+}$is open in $C(\Omega, S L(2, \mathbb{R}))$ and $\Lambda: \mathcal{U}(\Omega) \rightarrow \mathbb{R}$ is continuous.

This is essentially contained in Theorem 5 of [6] and its proof. Note, however, that there is a slight gap in the proof of that theorem in [6]: Its statement refers to arbitrary uniquely ergodic systems. But its proof makes crucial use of Theorem 4 of [6], which assumes not only unique ergodicity but also minimality. As far as the continuity statement goes, this gap can be bridged by restricting attention to a $T$-minimal subset $\Omega_{0}$ of $\Omega$. However, it does not seem to be clear that this yields the openess statement as well. Therefore, we conclude this section by noting that, given the methods provided in [6], one can base a proof of Theorem 5 on Theorem 4 above, similarly as the proof of Theorem 5 in [6] is based on Theorem 4 of [6].

\section{Acknowledgements}

The author gratefully acknowledges hospitality of the Edmund Landau Center at The Hebrew University, Jerusalem, where this work was started. He would particularly like to express his gratitude to H. Furstenberg, Y. Last and B. Weiss for stimulating discussions. Finally, he would like to thank the referee for his careful reading of the manuscript and various useful comments.

\section{References}

[1] G. Andre, S. Aubry, Analyticity breaking and Anderson localization in incommensurate lattices, Ann. Israel Phys. Soc. 3 (1980) 133-140.

[2] J. Avron, B. Simon, Singular continuous spectrum for a class of almost periodic Jacobi matrices, Bull. Amer. Math. Soc. 6 (1982) 81-85.

[3] R. Carmona, J. Lacroix, Spectral Theory of Random Schrödinger Operators, Birkhäuser, Boston, 1990.

[4] H.L. Cycon, R.G. Froese, W. Kirsch, B. Simon, Schrödinger Operators with Application to Quantum Mechanics and Global Geometry, Springer, Berlin, 1987.

[5] F. Durand, Linearly recurrent subshifts have a finite number of non-periodic subshift factors, Ergodic Theory Dynamical Systems 20 (2000) 1061-1078.

[6] A. Furman, On the multiplicative ergodic theorem for uniquely ergodic ergodic systems, Ann. Inst. Henri Poincaré Probab. Statist. 33 (1997) 797-815.

[7] H. Furstenberg, B. Weiss, Private communication.

[8] M.-R. Herman, Construction d'un difféomorphisme minimal d'entropie non nulle, Ergodic Theory Dynamical Systems 1 (1981) 65-76. 
[9] M.-R. Herman, Une méthode pour minorer les exposants de Lyapunov et quelques exemples montrant the caractère local d'un théorème d'Arnold et de Moser sur le tore de dimension 2, Comment. Math. Helv 58 (1983) 453-502.

[10] S. Jitomirskaya, Almost everything about the almost-Mathieu operator, II, in: XIth International Congress of Mathematical Physics (Paris, 1994), Internat. Press, Cambridge, MA, 1995, pp. 373-382.

[11] S. Jitomirskaya, Metal-insulator transition for the almost-Mathieu operator, Ann. of Math. (2) 150 (1999) 1159-1175.

[12] O. Knill, The upper Lyapunov exponent of $S L(2, \mathbb{R})$ cocycles: discontinuity and the problem of positivity, in: Lyapunov Exponents (Oberwolfach, 1990), in: Lecture Notes in Math., vol. 1486, Springer, Berlin, 1991, pp. 86-97.

[13] Y. Last, Almost everything about the almost-Mathieu operator, I, in: XIth International Congress of Mathematical Physics (Paris, 1994), Internat. Press, Cambridge, MA, 1995, pp. 373-382.

[14] Y. Last, B. Simon, Eigenfunctions, transfer matrices, and absolutely continuous spectrum for one-dimensional Schrödinger operators, Invent. Math. 135 (1999) 329-367.

[15] D. Lenz, Random operators and crossed products, Mathematical Physics Analysis and Geometry 2 (1999) 197-220.

[16] D. Lenz, Singular spectrum of Lebesgue measure zero for one-dimensional quasicrystals, Comm. Math. Phys. 227 (2002) 129-130.

[17] D. Lenz, Uniform ergodic theorems on subshifts over a finite alphabet, Ergodic Theory Dynamical Systems 22 (2002) $245-255$.

[18] D. Lenz, Hierarchical structures in Sturmian dynamical systems, Theoret. Comput. Sci. 303 (2003) 463-490.

[19] D. Ruelle, Ergodic theory of differentiable dynamical systems, Inst. Hautes Études Sci. Publ. Math. 50 (1979) $27-58$.

[20] W.A. Veech, Strict ergodicity in zero-dimensional dynamical systems and the Kronecker-Weyl theorem modulo 2, Trans. Amer. Math. Soc. 140 (1969) 1-33.

[21] P. Walters, Unique ergodicity and random matrix products, in: Lyapunov Exponents (Bremen, 1984), in: Lecture Notes in Math., vol. 1186, Springer, Berlin, 1986, pp. 37-55. 